\title{
Ultrasound guided paramedian approach compared with landmark based paramedian approach for thoracic epidural
}

\author{
Kashinath Khandu Jadhav ${ }^{1, *}$, Gita Nath ${ }^{2}$ \\ ${ }^{1}$ Assistant Professor, ${ }^{2}$ Consultant, Dept. of Anaesthesia, ${ }^{1}$ ACPM Medical College, Dhule, ${ }^{2}$ Global Hospital, Hyderabad, \\ Telangana, India
}

*Corresponding Author:

Email: kashinathj@yahoo.co.in

Received: $10^{\text {th }}$ June, 2017

Accepted: $14^{\text {th }}$ August, 2017

\begin{abstract}
Introduction: Currently, one has to rely on surface anatomical landmarks and loss of resistance technique for epidural analgesia. It frequently $(70 \%)$ leads to incorrect identification of a given inter-space as it is a blind technique. Therefore a better alternative technique is desirable.

Aims and Objectives: To evaluate and compare ultrasound guided thoracic epidural technique with landmark-based thoracic epidural technique via paramedian approach

Materials and Methods: A study was done on 34 patients in each group with age $\geq 20$ years, undergoing any major surgery, requiring thoracic epidural. The procedure and aim of the study was explained and a written informed consent was taken. Group 1 - (US group) - ultrasound guided thoracic epidural placement was done via paramedian approach .Group 2 - ((Landmark group) - landmark paramedian approach was done. The following parameters were studied - 1) Number of needle punctures and redirections through the same puncture site; 2) Number of attempts at catheter advancement; 3) Time taken for epidural catheter placement; 4) Pain felt by the patient during the procedure. The data was analysed using chi square test and student $t$ test.

Results: The number of needle punctures required, redirections and attempts of catheter advancement in both the groups were not statistically significant. The time required and pain felt for the procedure was significantly less in US Group as compared to Landmark group.

Conclusion: US guided thoracic epidural technique via paramedian approach took less time for the procedure and was associated with less pain as compared to landmark approach.
\end{abstract}

Keywords: Paramedian approach, Thoracic epidural, Ultrasound Guided.

\section{Introduction}

Epidural analgesia is a form of regional analgesia involving injection of drugs through a catheter placed in the epidural space. Epidural analgesia is considered the gold standard analgesic technique for major surgery. Advantages are attenuation of the stress response, beneficial effect on pulmonary mechanics, reduced incidence of postoperative myocardial infarction and attenuation of the hypercoagulable response to stress. ${ }^{1}$

The success of the technique depends on one's ability to accurately locate the epidural space. Currently, one has to rely on surface anatomical landmarks and loss of resistance to injection of saline or air. Anatomical landmarks are useful but are surrogate markers, difficult to palpate in obese patients and in those with edema over the back. They do not take into account anatomical variations or abnormalities and frequently (70\%) lead to incorrect identification of a given inter-space as it is a blind technique. ${ }^{2}$ When the puncture level is determined by palpation and compared with that determined by MRI, clinicians are correct in their assessment only in $30 \%$ of time. ${ }^{2}$ This may lead to multiple attempts at epidural needle placement, pain and discomfort to the patient, a failed block, complications, frustration for anesthesiologist and poor patient satisfaction. Therefore any alternative technique that can circumvent some of these shortcomings and facilitate localization of epidural space is desirable.

1. Recently, there has been an increased interest in the use of ultrasound (US) to guide peripheral nerve blocks and central neuraxial blocks (epidural or CSE), ${ }^{3-9}$ either to preview the anatomy before needle puncture or to visualize the advancing needle in real time. ${ }^{10}$ In a study involving obstetric patients, a preview scan performed before needle puncture improved the success rate of lumbar epidural access on the first attempt, reduced the number of puncture attempts, and the need to puncture at multiple levels. ${ }^{10}$ There are several features of the thoracic spine that distinguish it from the lumbar and cervical spine:

2. Downward angulation of the spinous processes of the thoracic vertebrae, particularly in the midthoracic region

3. The ligaments in this area are also less dense and a false loss of resistance is common

4. Less space between the spinous processes

5. Narrow spinal canal

6. Pressure in the epidural space is less negative than at the lumbar level. 
It is therefore technically more difficult to correctly identify the thoracic epidural space and much more common to encounter bony resistance during the placement of thoracic epidurals. Therefore chances of dural puncture and spinal cord injuries are also more. For this reason, many practitioners prefer to use a paramedian approach in this region.

Hence this study was done to evaluate and compare US guided thoracic epidural technique with landmarkbased thoracic epidural technique via paramedian approach.

\section{Aims and Objectives}

To evaluate and compare the ease of thoracic epidural insertion between ultrasound guided paramedian approach and landmark based paramedian approach by studying the following parameters:

1. Number of needle punctures and number of redirections through the same puncture site

2. Number of attempts of catheter advancement

3. Incidence of dural and intravascular puncture

4. Time taken for epidural catheter placement

5. Pain felt by the patient during the procedure, assessed by VAS score

\section{Material and Methods}

A Prospective randomized control study was done on 68 patients. These 68 patients were randomly allocated into two groups (34 patients in each group).

Group 1 (US Group): In this group US-guided paramedian thoracic epidural technique was used for thoracic epidural placement.

Group 2 (Landmark Group): In this group the landmark based paramedian approach was used for thoracic epidural placement.

Inclusion criteria: Patient aged $\geq 20$ years with ASA 1-4 status, undergoing any major surgery, requiring thoracic epidural for intraoperative and postoperative pain relief.

Exclusion criteria: Patients with coagulopathy, skin infection over the back and any neurological illness.

A written informed consent was taken from all patients after explaining the purpose and procedure of study. The visual analogue scale was also explained.

US group: In this group US-guided paramedian thoracic epidural technique was used for thoracic epidural placement using portable Micromaxx SONOSITE ultrasound machine. The thoracic spine of these patients were scanned preoperatively with curvilinear probe (frequency 3-5 Hz) in sitting position. The probe was placed vertically in the paramedian plane to visualize the ligamentum flavum and duramater as two parallel echogenic lines at a deeper level. The distance of the ligamentum flavum from the skin was measured using the built-in calipers in the US machine. Also the probe position was marked on the skin with a permanent marking pen both vertically and horizontally. The angle of the probe to the skin was noted visually. After preparing the skin with povidone iodine, local infiltration of $3 \mathrm{ml}$ of $2 \%$ lignocaine was given at the puncture site and then the epidural catheter placement was done under full aseptic precautions, without further US guidance. The skin puncture was done at the intersection of the vertical and horizontal lines and the needle was angled in the direction noted earlier.

Landmark group: In this group the landmark based paramedian approach was used for thoracic epidural placement. The skin was prepared with povidone iodine and local infiltration of $3 \mathrm{ml}$ of $2 \%$ lignocaine given at the puncture site and epidural catheter placement was done under full aseptic precautions in sitting position. At the thoracic level, 16 gauge epidural needle was advanced perpendicularly through the skin $1.0 \mathrm{~cm}$ lateral and inferior to the superior spinous process upto the lamina. The needle was then withdrawn approximately $2 \mathrm{~cm}$, redirected at a 15 to 20 -degree angle towards the midline and at 45-degree angle from the skin surface. Each time the bone came into contact the needle was withdrawn $0.5 \mathrm{~cm}$ to walk off the bone in medial or cephalad direction until the ligamentum flavum was entered.

The data analysis was done using chi square test, except for patients characteristics and time for epidural procedure, analysis was done using student $t$ test.

\section{Results}

Comparision of age, sex, height, weight, BMI showed no difference between the two groups, showing that they all were part of the same population (Table 1). There was no statistically significant difference in number of needle punctures (Table 2), number of redirections (Table 3) and total number of attempts of catheter advancement (Table 4) required for procedure between US group and Landmark group but there was a tendency of reduction in all the above mentioned parameters in US group as compared to landmark group. The VAS Score was statistically significantly decreased in US Group as compared to Landmark Group (Table 5). There was no incidence of dural puncture in US Group as well as in Landmark Group. There was statistically significant difference in the time required for the procedure between US Group and Landmark Group (Table 6). Ultrasound examination approximately required only 5 mins. 
Table 1: Comparision of Age, Height, Weight, BMI and Sex distribution between US group and Landmark group

\begin{tabular}{|l|c|c|c|c|}
\hline & US group & Landmark group & P value & Significance \\
\hline Age $($ years $) \pm$ SD & $47.05 \pm 14.72$ & $46.62 \pm 15.43$ & 0.901 & Not significant \\
\hline Sex $($ Male/Female) & $19 / 15$ & $20 / 14$ & $>0.1$ & Not significant \\
\hline Height $(\mathrm{cm}) \pm$ SD & $160.79 \pm 7.92$ & $162 \pm 6.85$ & 0.426 & Not significant \\
\hline Weight $(\mathrm{kg}) \pm$ SD & $65.14 \pm 12.15$ & $61.70 \pm 11.19$ & 0.229 & Not significant \\
\hline BMI $\left(\mathrm{kg} / \mathrm{m}^{2}\right) \pm$ SD & $25.11 \pm 3.80$ & $23.44 \pm 3.99$ & 0.082 & Not significant \\
\hline
\end{tabular}

Table 2: Comparison of No. of needle punctures, in US group and Landmark group

\begin{tabular}{|l|c|c|}
\hline Number Of Needle Punctures & US Group & Landmark Group \\
\hline 1 puncture & 27 & 23 \\
\hline 2 puncture & 7 & 9 \\
\hline 3 puncture & 0 & 2 \\
\hline NO. OF PATIENTS & 34 & 34 \\
\hline Chi $^{2}$ & \multicolumn{2}{|c|}{2.57} \\
\hline Df & \multicolumn{2}{|c|}{2} \\
\hline P Value & \multicolumn{2}{|c|}{ Not significant } \\
\hline Significance & \multicolumn{3}{|c|}{} \\
\hline
\end{tabular}

Table 3: Comparison of total number of redirections (NO. RED) between US group and Landmark group

\begin{tabular}{|l|c|c|}
\hline & US Group & Landmark Group \\
\hline No. of Patients & 34 & 34 \\
\hline No. Red & 87 & 92 \\
\hline Chi $^{2}$ & \multicolumn{3}{|c|}{0.139} \\
\hline df & \multicolumn{2}{|c|}{1} \\
\hline P Value & \multicolumn{2}{|c|}{$>0.5$} \\
\hline Significance & \multicolumn{3}{|c|}{ Not significant } \\
\hline
\end{tabular}

Table 4: Comparison of total number of attempts of catheter advancement (NO. AOCA) between US group and Landmark group

\begin{tabular}{|l|c|c|}
\hline & US group & Landmark group \\
\hline No. of Patients & 34 & 34 \\
\hline No. Aoca & 45 & 44 \\
\hline Chi $^{2}$ & \multicolumn{2}{|c|}{0.01} \\
\hline Df & \multicolumn{2}{|c|}{1} \\
\hline P Value & \multicolumn{2}{|c|}{$>0.8$} \\
\hline Significance & \multicolumn{2}{|c|}{ Not significant } \\
\hline
\end{tabular}

Table 5: Comparison of VAS score between US Group and Landmark Group

\begin{tabular}{|l|c|c|}
\hline \multicolumn{1}{|c|}{ VAS score } & US Group & Landmark Group \\
\hline 0 & 0 & 4 \\
\hline 1 & 2 & 0 \\
\hline 2 & 17 & 9 \\
\hline 3 & 14 & 6 \\
\hline 4 & 1 & 1 \\
\hline 5 & 0 & 1 \\
\hline 6 & 0 & 34 \\
\hline Total No. of Patients & 34 & \multicolumn{2}{|c|}{} \\
\hline Chi $^{2}$ & \multicolumn{2}{|c|}{ Significant } \\
\hline Df & \multicolumn{2}{|c|}{} \\
\hline P Value & \multicolumn{2}{|c|}{} \\
\hline Significance & \multicolumn{2}{|c|}{} \\
\hline
\end{tabular}


Table 6: Comparison of time required for the procedure (TFP) between US Group and Landmark Group

\begin{tabular}{|l|c|c|}
\hline & US Group & Landmark Group \\
\hline No. of Patients & 34 & 34 \\
\hline Mean of TFP & $10.62 \mathrm{~min}$ & $12.97 \mathrm{~min}$ \\
\hline S.D. & 3.1335 & 5.3057 \\
\hline t Value & \multicolumn{2}{|c|}{0.0302} \\
\hline P Value & \multicolumn{2}{|c|}{ Significant } \\
\hline Significance & \multicolumn{2}{|c|}{0.2261} \\
\hline
\end{tabular}

\section{Discussion}

We did not find any statistically significant difference in the number of needle punctures (Table 2), number of redirections (Table 3) and total number of attempts of catheter advancement (Table 4) required for procedure between US group and Landmark group but there was a tendency of reduction in all the above mentioned parameters in US group as compared to landmark group. Whereas $\mathrm{Grau}$ et $\mathrm{al}^{9}$ in their randomized, controlled study in 300 obstetric patients found a statistically significant decrease in the number of punctures, number of redirections and number of attempts in catheter advancement in Ultrasound guided technique as compared to landmark technique for lumbar epidural.

Rosaulian et $\mathrm{al}^{11}$ in their prospective cohort study in 20 patients using prepuncture US in paramedian approach for thoracic epidural placement found that the mean number of needle puncture was 1.3 , which is comparable to our figure of $1.21 \pm 0.41 \mathrm{In}$ another prospective cohort study done by Salman et $\mathrm{al}^{12}$ in 35 patients using prepuncture US in paramedian approach for thoracic epidural placement, found that the mean number of needle punctures required was 1 while in our study, the mean number of needle puncture was $1.2 \pm$ 0.4 which is quite comparable to their result. But in both these studies at the thoracic level "offset sagittal" or "paramedian sagittal" view was used. Moreover both these studies also differed from the present study as they were prospective cohort studies, without a control group, unlike the present randomized controlled study which attempted to assess the ease of insertion of thoracic epidural using US guidance.

Regarding pain during the procedure, we observed a statistically significant reduction in VAS score between the two groups (Table 5). Only one patient in US group had VAS score $>3$ as compared to 8 from landmark group. This reduction in VAS score may be due to the lower number of needle punctures and redirections, thus making the procedure less painful to the patients.

Thus when we compared the results of our study with those of the studies done at lumbar level ${ }^{9,13,14}$ our results matched with tendency to a reduction in the number of puncture attempts, number of redirections, number of attempts at catheter advancement, decreased VAS score and duration of procedure.

The time required for the thoracic epidural procedure in US group was statistically significantly reduced as compared to the Landmark group (Table 6). This indicates that US guided epidural insertion requires less time and is a quick procedure as compared to landmark procedure. The time taken for US examination was $4.97 \pm 0.97 \mathrm{~min}$ which means US examination takes only approximately 5 mins.

There was not a single incidence of dural or intravascular puncture during the procedure in both the groups.

Thus the use of prepuncture US for thoracic epidural in paramedian approach, definitely makes the procedure easier, less painful and quicker than landmark technique.

\section{Conclusion}

US guided thoracic epidurals took less time for the procedure and made the procedure quicker as compared to landmark technique. The US guided thoracic epidurals were associated with less pain, making procedure more comfortable for patients.

\section{References}

1. Nimmo SM. Benefit and outcome after epidural analgesia. Contin Educ Anaesth Crit Care Pain. 2004;4 (2):44-7.

2. Broadbent C, Maxwell W, Ferrie R, Wilson D, GauneCain M, Russell R. Ability of anaesthetists to identify a marked lumbar interspace. Anaesthesia. 2000; 55 (11): 1122-1126.

3. Marhofer P, Greher M, Kapral S. Ultrasound guidance in regional anaesthesia. British Journal of Anaesthesia. 2005;94(1):7-17.

4. Grau T , Leipold R, Conradi R, Martin E, Motsch J. Ultrasonography and epidural anaesthesia, Technical possibilities and boundaries of ultrasonic examination of the epidural space. Anaesthesist. 2001;50:94-101.

5. Grau T, Leipold R, Horter J, Conradi R, Martin E, Motsch J. Paramedian Access to the Epidural Space: The Optimum Window for Ultrasound Imaging. J Clin Anesthesia. 2001; 13: 213-217.

6. Grau T, Leipold R, Conradi R, Martin E, Motsch J. The lumbar epidural in - visualization by ultrasonography. British journal of Anesthesia. 2001;86(6):798-804.

7. Grau T, Leipold R, Conradi R, Martin E. Ultrasound control for presumed difficult epidural puncture. Acta Anaesthesiol Scand.2001; 45(6):766-71.

8. Grau T, Leipold R, Delorme S, Martin E, Motsch J. Ultrasound imaging of the thoracic epidural space. Reg Anesth Pain Med. 2002; 27(2): 200-206.

9. Grau T, Leipold R, Conradi R,Martin E, Motsch J. Efficacy of ultrasound imaging in obstetric epidural anesthesia. J. Clin. Anesthesia. 2002;14:170-5. 
10. Grau T, Leipold R, Fatehi S, Martin E, Motsch J. Realtime ultrasonic observation of combined spinal-epidural anaesthesia. European Journal of Anaesthesiology. 2004;21(1):25-31.

11. Rasoulian A, Lohser J, Najafi M, Rafii-Tari H, Tran D, Kamani AA. Utility of prepuncture ultrasound for localization of the thoracic epidural space. Can J Anaesth. 2011;58(9):815-23.

12. Salman A, Arzola C, Tharmaratnam U, Balki M. Ultrasound imaging of the thoracic spine in paramedian sagittal oblique plane: the correlation between estimated and actual depth to the epidural space. Reg Anesth Pain Med.2011;36(6):542-7.

13. Arzola C, Davies S, Rofaeel A, Carvalho JCA.

Ultrasound using the transverse approach to the lumbar spine provides reliable landmarks for labor epidurals. Anesth Analg .2007;104(5):1188-92.

14. Balki M, Lee Y, Halpern S, Carvalho JCA. Ultrasound imaging of the lumbar spine in the transverse plane: the correlation between estimated and actual depth to the epidural space in obese parturients. Anesth Analg. 2009;108:1876-81. 